\title{
Exercise interventions for cognitive function in adults older than 50: a systematic review with meta-analysis
}

\author{
Joseph Michael Northey, ${ }^{1,2}$ Nicolas Cherbuin, ${ }^{3}$ Kate Louise Pumpa, ${ }^{1,2}$ Disa Jane Smee, ${ }^{2}$ \\ Ben Rattray ${ }^{1,2}$
}

- Additional material is published online only. To view please visit the journal online (http://dx.doi.org/10.1136/ bjsports-2016-096587).

${ }^{1} U C$ Research Institute for Sport and Exercise (UCRISE), University of Canberra, Canberra, Australia

${ }^{2}$ Discipline of Sport and Exercise Science, Faculty of Health, University of Canberra, Canberra, Australia

${ }^{3}$ Centre for Research on Ageing, Health and Wellbeing, Australian National University, Canberra, Australia

\section{Correspondence to}

Joseph Michael Northey, Joseph Northey, University of Canberra Research Institute for Sport and Exercise, Building 29, University Drive, Bruce ACT, 2617, Australia;

joe.northey@canberra.edu.au

Accepted 12 March 2017 Published Online First 24 April 2017
Check for updates

To cite: Northey JM, Cherbuin N, Pumpa KL, et al. Br I Sports Med 2018:52:154-160.

\section{ABSTRACT}

Background Physical exercise is seen as a promising intervention to prevent or delay cognitive decline in individuals aged 50 years and older, yet the evidence from reviews is not conclusive.

Objectives To determine if physical exercise is effective in improving cognitive function in this population.

Design Systematic review with multilevel meta-analysis. Data sources Electronic databases Medline (PubMed), EMBASE (Scopus), PsychINFO and CENTRAL (Cochrane) from inception to November 2016.

Eligibility criteria Randomised controlled trials of physical exercise interventions in community-dwelling adults older than 50 years, with an outcome measure of cognitive function.

Results The search returned 12820 records, of which 39 studies were included in the systematic review. Analysis of 333 dependent effect sizes from 36 studies showed that physical exercise improved cognitive function $(0.29 ; 95 \% \mathrm{Cl} 0.17$ to $0.41 ; p<0.01)$. Interventions of aerobic exercise, resistance training, multicomponent training and tai chi, all had significant point estimates. When exercise prescription was examined, a duration of 45-60 min per session and at least moderate intensity, were associated with benefits to cognition. The results of the meta-analysis were consistent and independent of the cognitive domain tested or the cognitive status of the participants. Conclusions Physical exercise improved cognitive function in the over 50s, regardless of the cognitive status of participants. To improve cognitive function, this meta-analysis provides clinicians with evidence to recommend that patients obtain both aerobic and resistance exercise of at least moderate intensity on as many days of the week as feasible, in line with current exercise guidelines.

\section{INTRODUCTION}

Physical exercise shows promise as a modifiable risk factor to reduce the risk of dementia and related neurodegenerative diseases. ${ }^{1}$ As cognitive function declines with advancing age, a physically active lifestyle has an important role in reducing such declines, ${ }^{23}$ as well as the incidence of dementia. ${ }^{4}$ It is hypothesised that the neural and vascular adaptations to physical exercise improve cognitive function through promotion of neurogenesis, angiogenesis, synaptic plasticity, decreased proinflammatory processes and reduced cellular damage due to oxidative stress. ${ }^{5}$ While lifelong participation in physical exercise may be preferable, the adoption of exercise at any age to delay or reverse cognitive decline is worthwhile given the prevalence of physical inactivity and the increasing proportion of older adults in the population.

Although early meta-analyses, such as a study of aerobic exercise interventions, ${ }^{6}$ showed large benefits to cognitive function in older adults, more recent systematic reviews ${ }^{7}$ and meta-analytical studies $^{8-10}$ are much less conclusive. For example, a recent meta-analysis of aerobic, resistance training and tai chi interventions in people older than 50 showed little benefit of exercise on cognitive function. ${ }^{9}$ The discrepancy in findings is partly because existing reviews are excessively restrictive in their inclusion criteria, often considering only one mode of exercise (eg, recent reviews of aerobic training only ${ }^{10}{ }^{11}$ ) or a narrow range of publication years. Thus, the numerous meta-analyses published provide incomplete summaries of the available evidence in people aged 50 and over. Studies which prescribe a combination of both aerobic and resistance training components in one intervention (here on called multicomponent training) have not been reviewed in healthy older adults since the 2001 study of Colcombe and $\mathrm{Kramer}^{6}$ despite global guidelines recommending this type of training in older adults. ${ }^{12} 13$ Alternative modes of exercise such as yoga ${ }^{14}$ or tai chi $^{15}$ may also be beneficial to cognitive function, yet randomised controlled trials (RCTs) of these modes in older adults have not been specifically reviewed. Importantly, prior reviews offer relatively little information about the optimal prescription of physical exercise for cognitive health. Physical exercise provides a complex stimulus for adaptation in the body and its dosage can be modulated by various parameters, including duration, frequency, intensity and the mode or type of exercise. Despite this, many reviews do not take into account the importance of exercise prescription variables in either the analysis or discussion of the literature. Consequently, there is an urgent need for guidelines on the type or amount of exercise a clinician should recommend to their patient.

To deal with these research gaps, we have completed a comprehensive meta-analysis which includes a larger number of studies by imposing no limit on publication date or exercise mode. This study examines four key issues including: (1) the effects of supervised exercise interventions of aerobic, resistance, multicomponent, tai chi and yoga training modes on cognitive function; (2) the influence of exercise training variables, including the duration, frequency, intensity and length of exercise; (3) the differentiation of exercise effects on global cognition and domains of cognition, including attention, executive function, memory working memory; and (4) the impact of study 
design, including the nature of the control group and the baseline cognitive status of participants.

\section{METHODS}

This systematic review with meta-analysis was conducted in accordance with established guidelines from Preferred Reporting Items for Systematic Reviews and Meta-Analysis (PRISMA). ${ }^{16}$

\section{Search strategy}

To direct the design of the search strategy, previously completed meta-analyses ${ }^{6-9}$ were reviewed and search terms were piloted to ensure a comprehensive identification of potential articles for inclusion in the systematic review. A computer search of Medline (PubMed), EMBASE (Scopus), PsychINFO and CENTRAL (Cochrane Central Register of Controlled Trials) was then conducted up to November 2016, using medical subject headings (MeSH) for 'exercise' and 'cognition' (box 1). 'Exercise' and 'cognition' search terms were combined with 'AND' and searched in 'All Fields' with the limits human and English language (see online supplementary table A). All returned titles were screened by the first author (JMN) to exclude duplicate or clearly non-relevant studies. The abstract of each remaining study was then independently reviewed by JMN and BR. The preceding stages were overinclusive. Subsequently, the full texts of the remaining studies were independently reviewed by JMN, KLP and DJS against the inclusion and exclusion criteria. Disagreements were discussed and consensus reached among the authors in all cases. Review authors subsequently searched the bibliography of included articles and prior reviews to ensure that relevant articles had been captured by the search strategy. All studies in the systematic review were eligible for inclusion in the meta-analysis.

\section{Inclusion and exclusion criteria}

Studies were included from the initial search if they strictly met the following criteria: (1) studies of community dwelling men or women aged 50 years or older. Because criteria for diagnosing cognitive ability (eg, the presence of mild cognitive impairment (MCI)) differ between studies and prior reviews, ${ }^{8}$ there were no limitations on baseline cognitive status. However, studies which included clinical samples with other neurological (eg, stroke) or mental illnesses (eg, depression) were excluded. (2) A structured

\section{Box 1 Search terms for exercise and cognition}

Exercise search terms combined with 'OR'
Exercise
Aerobic exercise
Plyometric exercise
Resistance training
Strength training
$\quad$ Muscle stretching exercises
Physical conditioning, human
Walking
Tai ji (tai chi)
Yoga
Cognition search terms combined with 'OR'
Cognition
Memory
Executive function
Dementia
Alzheimer disease

exercise programme of any mode, duration, frequency or intensity. Exercise programmes that were not explicitly stated as fully supervised, or of $<4$ weeks, were excluded. Studies must have allowed the isolated effects of exercise to be measured. (3) A control group could include no contact, waiting list, attention control, sham exercise or alternative active treatment. (4) At least one outcome measure of cognition, measured at baseline and follow-up by any validated neuropsychological test of cognition. (5) The study design was strictly limited to RCTs. (6) A trial must have been published in a peer-reviewed journal.

\section{Data extraction}

Data on the study population, intervention, control group and outcome measures were independently extracted into a standardised form by two review authors (JMN and DJS). Where available, the mean change from baseline, the SD of the mean change and the number of participants at each assessment for all groups were extracted. Where authors reported more than one measured time-point throughout an intervention, only the longest follow-up period in which the exercise intervention was continued was admitted. Data were extracted such that an improvement in performance was coded as a positive change score. Variance information was converted to SD. Where the mean change from baseline was not available, it was calculated from baseline and postintervention cognition scores. Similarly, where change from baseline SD was not available, it was calculated from baseline SD and postintervention SD using the formula: $\mathrm{SD}_{\text {change }}=\sqrt{ }\left(\mathrm{SD}_{\text {baseline }}^{2}+\mathrm{SD}^{2}\right.$ postintervention $)-(2 \times$ Corr $\times$ $\mathrm{SD}^{2}$ baseline $\times \mathrm{SD}^{2}$ postintervention $)$, where Corr $=0.5$. The value for Corr was imputed on the assumption of a moderate correlation between baseline and postintervention measures. While this may overestimate the change from baseline SD, it represents a conservative approach that is consistent with previous meta-analyses. ${ }^{8}$ Sensitivity analysis was performed to assess the impact of including SDs calculated from the imputed Corr value. Unpublished data (mean and SD) were requested from the authors of four studies, ${ }^{17-20}$ and clarification of published data was requested from the author of one study. ${ }^{21}$ Subsequently, unpublished data from Langlois et $a l^{18}$ and Tsai et $a l^{20}$ were included in the quantitative assessment.

\section{Data coding}

Descriptive data from each study were coded for inclusion in the moderator analysis (described later).

\section{Exercise moderators}

In accordance with objective 2 of the review, the characteristics of the physical exercise intervention were coded into categorical variables. First, the mode of exercise was classified as aerobic, resistance training, multicomponent training (an intervention with both aerobic and resistance training components), tai chi or yoga. Exercise intensity (low; moderate; high) was coded in reference to published guidelines that reconcile differences in the terminology used to describe exercise intensity. ${ }^{22} 23$ The duration (minutes each session lasted, including any warm-up or cool-down) and length (weeks of exercise: short: 4-12 weeks; medium: 13-26 weeks; long: > 26 weeks) of the training period in the exercise and control groups were coded using a prior review as a guide. ${ }^{6}$ The coding for duration (short: $\leq 45 \mathrm{~min}$; medium: $>45$ to $\leq 60 \mathrm{~min}$; long: $>60 \mathrm{~min}$ ) was modified from Colcombe and Kramer, ${ }^{6}$ who defined a 'short' duration as $\leq 30$ min. The current review modified this cut-off point as it did not include any studies which prescribed exercise for $\leq 30 \mathrm{~min}$, 
possibly owing to differences in the inclusion criteria. In addition, the frequency (number of exercise sessions per week: low: $\leq 2$; medium: $3-4$; high: $5-7$ ) of both groups was coded.

\section{Cognitive moderators}

To address objective number 3, neuropsychological tests were classified according to the domain of cognition being assessed, similar to previous reviews. ${ }^{24}$ The domains considered were global cognition (eg, The Mini-Mental State Examination), attention (sustained alertness, including the ability to process information rapidly), executive function (a set of cognitive processes responsible for the initiation and monitoring of goal-orientated behaviours), memory (storage and retrieval of information) and working memory (short-term manipulation of encountered information). ${ }^{24}$

\section{Study design moderators}

Characteristics of the control group and the baseline cognitive status of the participants were categorised in accordance with objective 4 . The control group of each study was organised into four categories, including no contact (eg, instructed to maintain current lifestyle), active (eg, sham exercise intervention such as stretching), educational (eg, health lectures or a computer course) or social (eg, social meeting groups). Finally, the baseline cognitive status of participants was coded with respect to the presence of MCI (yes, no or unclear) using the criteria for MCI adopted by Gates et al. ${ }^{8}$

\section{Risk of bias assessment}

Two authors (JMN and DJS) independently assessed the risk of bias at the study level of included RCTs in accordance with the Cochrane Collaboration Guidelines. ${ }^{25}$ The risk of bias was assessed as being 'low', 'high' or 'unclear' across the following domains: randomisation; allocation concealment; blinding of therapists (intervention supervisors); blinding of participants; blinding of outcome assessors; handling of incomplete data (use of intention-to-treat analysis); selective reporting and any other risk of bias. Discrepancies in the risk of bias assessment were resolved by discussion among review authors.

\section{Statistical analysis}

Statistical analysis was conducted with $\mathrm{R}$ version $3.2 .1^{26}$ using the metafor package 1.9.7. ${ }^{27}$

\section{Meta-analysis}

The standardised mean difference (SMD) was calculated using the mean change from baseline, change SD and number of participants for the exercise and control groups. To account for dependency between effect sizes, a multilevel random-effects model was run using a restricted maximum likelihood estimator. ${ }^{28}$ Unlike traditional meta-analysis, which requires independence of effect sizes, the use of a multilevel meta-analysis accounted for dependency by fitting the term study as a random factor in the model. The multilevel model was used to estimate an overall effect size of exercise interventions versus control on cognitive function (objective 1). Heterogeneity was assessed using the $\mathrm{Q}$ statistic (with $\mathrm{p}<0.10$ suggesting statistically significant heterogeneity). ${ }^{29} \mathrm{~A}$ mixed-effects model was fitted to examine the moderators described above as potential sources of variance. Initially, separate models were fitted to determine the main effects for each exercise (objective 2), cognitive (objective 3 ) and study design (objective 4) moderator. The analysis of main effects was interpreted using the $95 \%$ CI for the point estimates of each level of a moderator and the statistical significance of the omnibus test. Following analysis of main effects, where appropriate, and in accordance with the purpose of the study, moderators found to be significant were added to one model in order to examine possible confounding and to test for interaction effects. ${ }^{28}$

Funnel plots of the effect size against the SE of the effect size were visually inspected for small-sample bias and Egger's test values with 95\% CI for funnel plot asymmetry were calculated. $^{30}$ To run Egger's test, the multilevel random-effects model was modified to include the SE of the effect size as a moderator. ${ }^{31}$ Small-sample bias was considered to be present when the funnel plot appeared asymmetrical and the intercept of the Egger's test was significantly different from zero $(\mathrm{p}<0.10){ }^{3032}$

The GRADE guidelines ${ }^{33}$ were applied independently by two review authors (JMN and DJS) to evaluate the overall quality of evidence for the comparison of exercise versus control groups for cognitive function. The overall quality of evidence was initially considered 'high' owing to the inclusion criteria, which stipulated that studies must be a RCT. The quality of evidence was downgraded by one level where each of the following four factors applied: (1) high risk of study bias; (2) inconsistency evaluated by a substantial $\mathrm{I}^{2}(>75 \%)$. $\mathrm{I}^{2}$ was calculated with the following formula: $\left.\mathrm{I}^{2}(\%)=(\mathrm{Q}-\mathrm{df}) / \mathrm{Q}\right) \times 100$; (3) imprecision due to fewer than 400 participants in the outcome and (4) publication bias identified through an evaluation of funnel plot asymmetry. Indirectness was not considered owing to the inclusion criteria of the review.

\section{RESULTS}

The flow of records through the review is summarised in figure 1. The initial search strategy returned 12820 records, of which 215 were retrieved for full-text review. Forty-three articles met the criteria for inclusion in the qualitative analysis. The analysis was conducted on each study. Subsequently, on four occasions when a study produced more than one publication (Blumenthal et $a l^{34}$ and Madden $e t a l^{35}$; Liu-Ambrose $e t$ al $^{36}$ and Liu-Ambrose et $a l^{37}$; Nagamatsu et $a l^{38}$ and ten Brinke et $a l^{39}$; and Erickson et $a l^{40}$ and Voss et $a l^{41}$ ), they were considered as one study for analysis. Finally, 39 studies were included in the qualitative analysis, with all studies eligible for inclusion in the quantitative analysis. The exercise mode was used to group the studies into interventions of aerobic exercise $(\mathrm{k}=18),{ }^{17} 19343538-52$ resistance training $(\mathrm{k}=13),{ }^{20} 21$ 36-39 47 53-59 multicomponent training $(\mathrm{k}=10),{ }^{18} 57$ 60-66 tai chi $(\mathrm{k}=4)^{4667-69}$ and yoga $(\mathrm{k}=2) .{ }^{3470}$ The methodological characteristics of these studies are summarised and available in supplementary table B.

\section{Risk of bias}

The risk of bias assessment is summarised in figure 2 . The majority of studies did not describe the process of sequence generation or allocation concealment in sufficient detail and were judged as having 'unclear' risk of bias for these domains. In all of the assessed studies it was neither practical nor possible to blind the participants and therapists. It was judged that this presented a low risk of bias for the therapists but a high risk of bias for the participants. Studies were judged to have a high risk of bias for incomplete outcome data if they did not employ intention-totreat principles in the data analysis or did not account for drop outs. All other domains were judged to have a low to unclear risk of bias. 


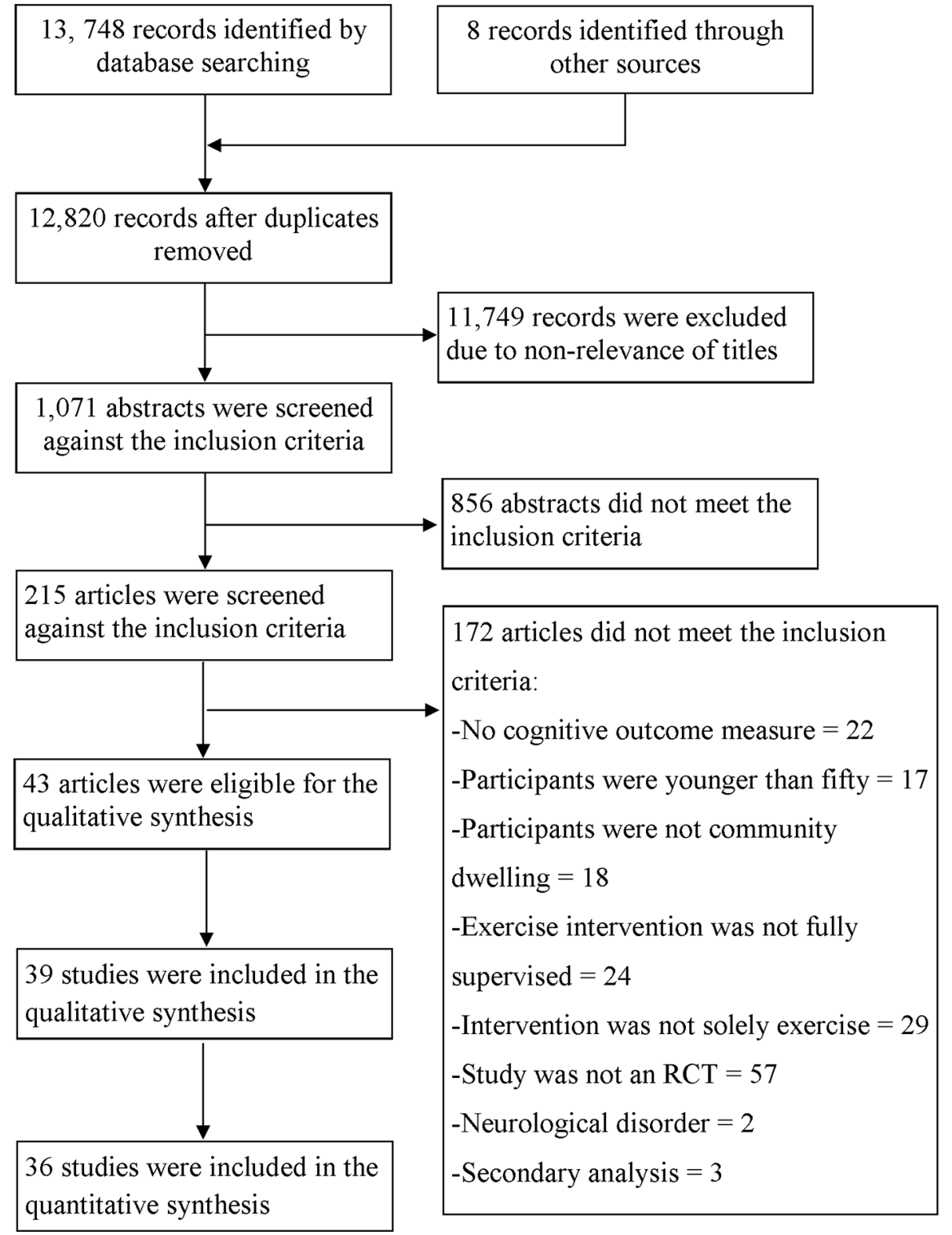

Figure 1 PRISMA (Preferred Reporting Items for Systematic Reviews and Meta-Analysis) flow diagram of each stage of the study selection. RCT, randomised controlled trial.

\section{Effect of exercise on cognition}

The multilevel meta-analysis provides an estimate of the difference between physical exercise and control on cognitive function for 333 dependent effect sizes across 36 studies. The SMD was 0.29 (95\% CI 0.17 to $0.41 ; \mathrm{p}<0.01)$ and there was significant heterogeneity present $\left(\mathrm{Q}_{332}=811.00 ; \mathrm{p}<0.01\right)$. The funnel plot of included studies is presented in figure 3 . Visual inspection of the funnel plot and a non-significant Egger's regression intercept $(p=0.175)$ suggests the absence of funnel plot asymmetry.

According to the GRADE guidelines, the evidence for this outcome was classified as moderate quality. The evidence was conservatively downgraded owing to the level of uncertainty across each domain of the risk of bias tool (figure 3 ).

\section{Moderator analysis}

To investigate potential sources of variance, moderators were analysed one at a time in separate models. The results from this stage of analysis are summarised in table 1 and described below.

\section{Exercise moderators}

When exercise mode was examined as a moderator, all modes of exercise produced significant $(\mathrm{p}<0.01)$ and positive effect estimates, except for yoga $(p=0.27)$. Studies where the duration of exercise was medium ( $>45 \mathrm{~min}$ to $\leq 60 \mathrm{~min}$; $\mathrm{p}<0.01$ ) were associated with significant estimates in comparison with short- and long-duration exercise, which were not statistically significant. Moderate $(p=0.02)$ and vigorous $(p<0.01)$ intensities had similar sized effect estimates, whereas low intensity was not significant $(p=0.11)$. The frequency and length of the exercise intervention produced significant estimates across all three categories. For each exercise moderator analysis, the omnibus test was significant and the 95\% CI for each factor overlapped.

\section{Cognitive moderators}

The effect of exercise on cognition was statistically significant for all domains, except global cognition. As prior reviews have indicated the effects of exercise on cognition may vary 


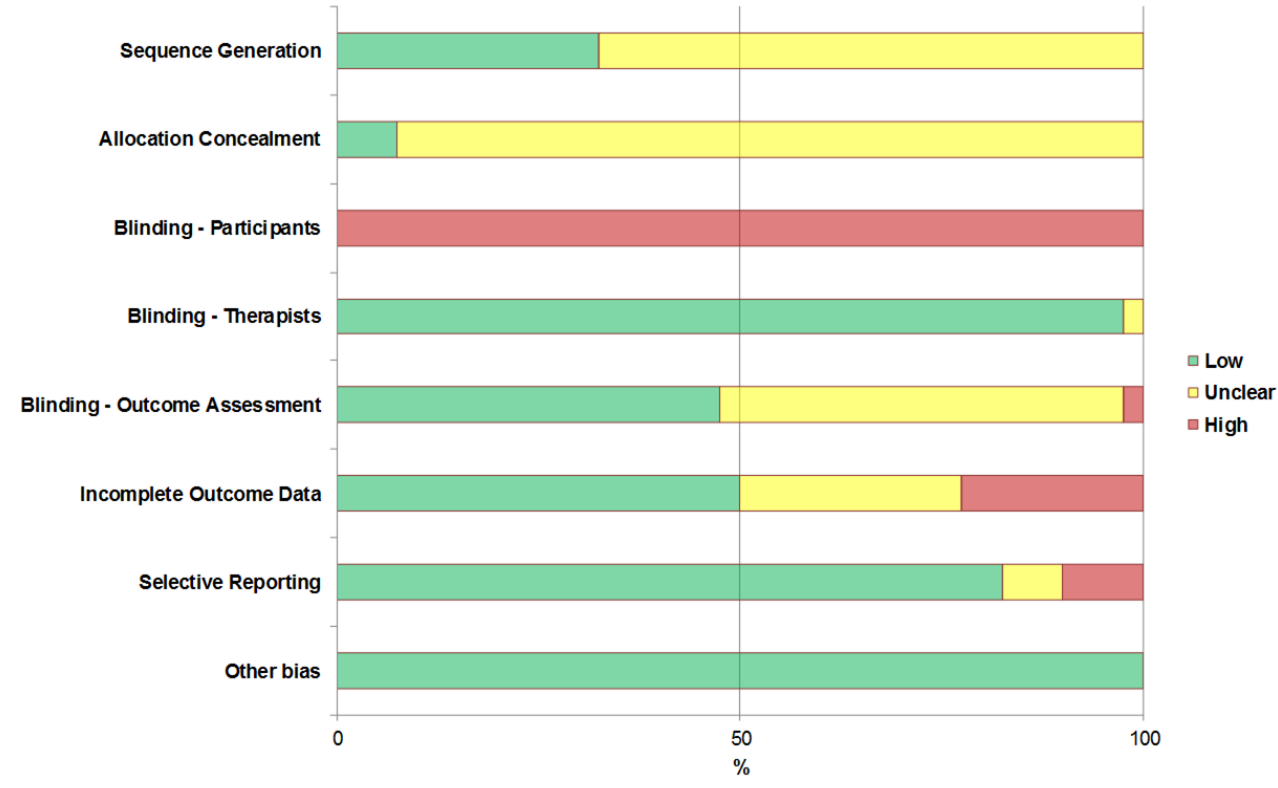

Figure 2 Analysis of the risk of bias in included studies in accordance with the Cochrane Collaboration guidelines.

depending on the mode of exercise and cognitive domain, we included both these moderators as an interaction term in a separate model. Studies of resistance training had significant interaction effects on executive function $(\mathrm{SMD}=0.49,95 \% \mathrm{CI} 0.20$ to $0.78 ; \mathrm{p}<0.01)$, memory $(\mathrm{SMD}=0.54,95 \% \mathrm{CI} 0.23$ to 0.85 ; $\mathrm{p}<0.01)$ and working memory $(\mathrm{SMD}=0.49$, 95\% CI 0.16 to $0.82 ; \mathrm{p}<0.01)$. There was also a significant tai chi $\mathrm{x}$ working memory interaction $(\mathrm{SMD}=-0.70,95 \% \mathrm{CI}-1.21$ to -0.19 ; $\mathrm{p}=0.01)$. All other interaction terms were non-significant.

\section{Study design moderators}

The type of control group was associated with differences in the statistical significance of the effect size estimated. When the control group involved either no contact (eg, waiting list, usual care; $\mathrm{p}<0.01$ ) or education (eg, computer course, health lectures; $\mathrm{p}=0.01$ ) the estimate was statistically significant. Where the control condition was exposed to an active control (eg, stretching; $\mathrm{p}=0.17)$ or social group $(\mathrm{p}=0.62)$, the effect

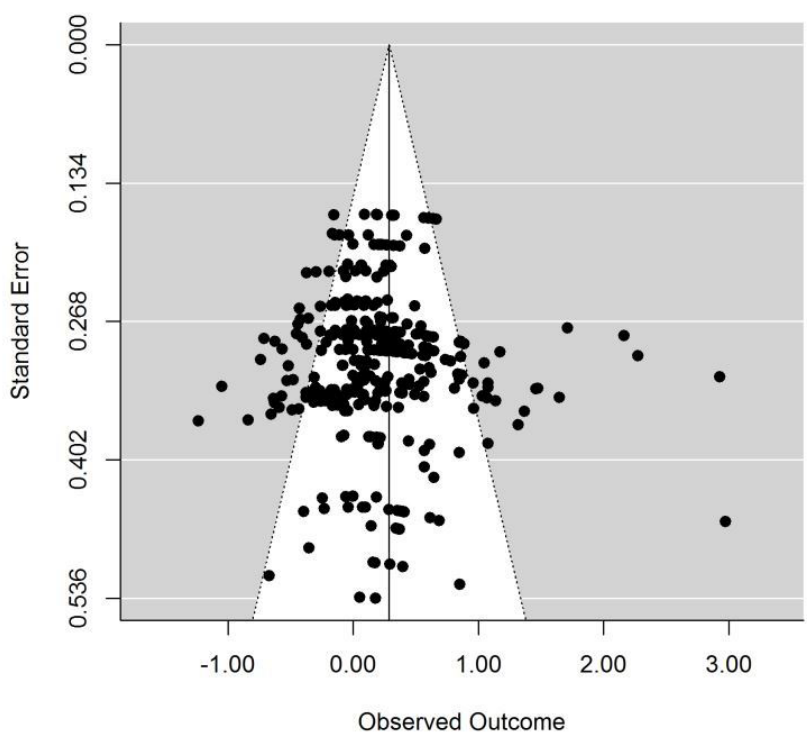

Figure 3 Funnel plot of included dependent effect sizes ( $k=333)$. size was still positive but no longer statistically significant. As shown in table 1 , the cognitive status of the study participants did not change the overall result of the meta-analysis. To test the differential effect of exercise on cognitive domain dependent on cognitive status, both moderators were entered with an interaction effect. There were no significant cognitive status $\mathrm{x}$ domain interaction effects. In addition, cognitive status and exercise mode were entered into a separate model with an interaction term. There was only one significant interaction effect for tai chi $\mathrm{x}$ unclear $(\mathrm{SMD}=1.11,95 \% \mathrm{CI} 0.16$ to $2.07 ; \mathrm{p}=0.02)$

\section{DISCUSSION}

This study conducted the most comprehensive systematic review of RCTs in adults $>50$ years of age to date. Importantly, it did not limit the inclusion of studies by exercise mode or publication date and incorporated a multilevel meta-analysis method that included exploration of moderator variables and formal assessment of small-study effects. The key finding from this study is that physical exercise interventions are effective in improving cognitive function in adults aged $>50$ years, regardless of cognitive status.

\section{Exercise mode}

Of the traditional modes of exercise, studies incorporating a component of aerobic or resistance training showed similar effect size estimates. Aerobic exercise has previously been associated with large improvements in complex cognitive tasks such as executive function. ${ }^{6}$ Although the size of the effect estimates reported here is smaller than reported by Colcombe et al, ${ }^{6}$ our study also suggests that aerobic exercise is beneficial to the cognitive functioning of older adults. This finding is of importance as the results of more recent reviews ${ }^{8-10}$ collectively provided little evidence of aerobic training benefits and contradicted exercise recommendations for this age group. An important feature differentiating this study from more recent reviews of aerobic exercise is the multilevel analysis model used and the absence of restrictions on publication date. As a result of these differences, this review was able to conduct a robust investigation, with greater statistical power and included a large number of otherwise relevant studies not eligible for these recent reviews. 
Table 1 Results of moderator analysis

\begin{tabular}{|c|c|c|c|c|}
\hline Moderator & No. of effect sizes & Estimate Mean $(95 \% \mathrm{Cl})$ & Q statistic & Omnibus test of moderators \\
\hline \multicolumn{5}{|l|}{ Exercise moderators } \\
\hline Mode & & & $Q_{328}=781.68 ; p<0.01$ & $Q_{5}=39.53 ; p<0.01$ \\
\hline Aerobic & 153 & 0.24 (0.10 to 0.37 ) & & \\
\hline Resistance training & 80 & 0.29 (0.13 to 0.44$)$ & & \\
\hline Multicomponent training & 47 & 0.33 (0.14 to 0.53$)$ & & \\
\hline Tai chi & 25 & 0.52 (0.32 to 0.71$)$ & & \\
\hline Yoga & 28 & $0.13(-0.10$ to 0.36$)$ & & \\
\hline Duration & & & $Q_{318}=789.68 ; p<0.01$ & $Q_{3}=27.83 ; p<0.01$ \\
\hline Short ( $\leq 45 \mathrm{~min})$ & 36 & $0.09(-0.28$ to 0.46$)$ & & \\
\hline Medium ( $>45$ to $\leq 60 \mathrm{~min}$ ) & 263 & 0.31 (0.16 to 0.46 ) & & \\
\hline Long (>60 min) & 24 & $0.33(-0.04$ to 0.65$)$ & & \\
\hline Frequency & & & $Q_{329}=804.58 ; p<0.01$ & $Q_{3}=24.12 ; p<0.01$ \\
\hline $\operatorname{Low}(\leq 2)$ & 92 & 0.32 (0.13 to 0.52 ) & & \\
\hline Medium (3-4) & 229 & 0.24 (0.07 to 0.40$)$ & & \\
\hline High (5--7) & 13 & 0.69 (0.10 to 1.28$)$ & & \\
\hline Intensity & & & $Q_{207}=264.61 ; p<0.01$ & $Q_{3}=13.55 ; p<0.01$ \\
\hline Low & 71 & $0.10(-0.02$ to 0.23$)$ & & \\
\hline Moderate & 57 & 0.17 (0.03 to 0.33$)$ & & \\
\hline High & 83 & 0.16 (0.04 to 0.27$)$ & & \\
\hline Length & & & $\mathrm{Q}_{330}=807.48 \mathrm{P}<0.01$ & $Q_{3}=23.32 ; p<0.01$ \\
\hline Short (4-12 weeks) & 78 & 0.31 (0.09 to 0.54$)$ & & \\
\hline Medium (13-26 weeks) & 170 & 0.28 (0.10 to 0.47$)$ & & \\
\hline Long (>26 weeks) & 86 & 0.27 (0.03 to 0.52$)$ & & \\
\hline \multicolumn{5}{|l|}{ Cognitive moderators } \\
\hline Cognitive domain & & & $Q_{303}=795.06 ; p<0.01$ & $Q_{5}=28.08 ; p<0.01$ \\
\hline Global cognition & 6 & $0.16(-0.14$ to 0.47$)$ & & \\
\hline Attention & 87 & 0.27 (0.14 to 0.41$)$ & & \\
\hline Executive function & 94 & 0.34 (0.20 to 0.47 ) & & \\
\hline Memory & 81 & $0.36(0.22$ to 0.50$)$ & & \\
\hline Working memory & 36 & $0.29(0.12$ to 0.45$)$ & & \\
\hline \multicolumn{5}{|l|}{ Study design moderators } \\
\hline Control group & & & $Q_{328}=785.37 ; p<0.01$ & $Q_{4}=25.52 ; p<0.01$ \\
\hline Active & 120 & $0.13(-0.06$ to 0.32$)$ & & \\
\hline Education & 17 & 0.48 (0.14 to 0.82 ) & & \\
\hline No contact & 189 & 0.34 (0.17 to 0.51 ) & & \\
\hline Social & 7 & $0.20(-0.58$ to 0.98$)$ & & \\
\hline Cognitive status & & & $Q_{330}=797.56 ; p<0.01$ & $Q_{3}=21.62 ; p<0.01$ \\
\hline $\mathrm{MCl}-$ Yes & 197 & 0.28 (0.11 to 0.44$)$ & & \\
\hline $\mathrm{MCl}$ - No & 41 & 0.36 (0.04 to 0.68$)$ & & \\
\hline Unclear & 96 & 0.28 (0.05 to 0.51$)$ & & \\
\hline
\end{tabular}

$\mathrm{MCl}$, mild cognitive impairment.

This study confirms previous suggestions that resistance training may play an important role in improving cognitive function in older adults. ${ }^{8}$ The moderator analysis showed significant interaction effects for resistance training with executive function, memory and working memory. Although this does not show that resistance training is better than other modes of exercise, it does suggest that this type of training has particularly pronounced effects on these domains of cognitive function. In contrast to our results, a previous meta-analysis which tested the effect of resistance training found no benefit to cognition in older adults. ${ }^{9}$ However, our review contained a greater number of resistance training trials than the previous meta-analysis ${ }^{9}$ by including studies published before $2002^{4756}$ and those published since their census date in 2012. ${ }^{37-39}$ 57-59 Although prior reviews showed that the addition of resistance training to an aerobic intervention may have additional benefits to cognition compared with aerobic exercise alone, ${ }^{624}$ this is the first review to specifically investigate the effect of multicomponent training on cognitive function in this age group. As exercise guidelines for this age group recommend obtaining both aerobic exercise and resistance training to improve health and reduce the risk of disease, ${ }^{12}{ }^{13}$ it was important for this type of intervention to be reviewed for its effect on cognition. Our meta-analysis provides positive evidence for the prescription of both aerobic and resistance training (ie, multicomponent training), in accordance with exercise recommendations, for this age group to specifically improve cognitive functions.

Our meta-analysis also showed that tai chi improved cognitive function in this age group, as suggested in previous studies. ${ }^{15}$ However, this finding should be considered in the context of the small number of tai chi studies included in this review. Further evidence is required from large well-designed RCTs to confirm this effect. Nevertheless, it is an important finding because non-traditional modes of exercise, such as tai chi, may be suitable for less functional populations. 


\section{Exercise prescription}

An important objective of this review was to investigate the role of physical exercise training principles on changes in cognitive function. The moderator analysis in this study showed that exercise of between 45 and $60 \mathrm{~min}$ in duration, of moderate or vigorous intensity and of any frequency or length is beneficial to cognitive function. Although the moderator analysis suggested statistical differences in the effect estimates between levels of each moderator, the overlapping $95 \%$ CIs made it difficult to discern the practical significance of these differences. While there are statistical methods to identify these differences (namely pairwise comparisons), there is a risk of inflated type I errors and the possibility of false-positive or false-negative results with the application of correction factors. As this review provides evidence of the beneficial effect of physical exercise on cognitive function, future RCTs must instead move beyond investigating effectiveness and begin to refine the prescription of training to promote the greatest benefits to cognitive function.

\section{Trial design moderators}

Several trial design characteristics were investigated as moderating factors. First, the presence of MCI in study participants did not change the overall findings of the meta-analysis. This is important owing to the increased risk of transitioning to a diagnosis of Alzheimer's disease or dementia with the presence of MCI. ${ }^{72}$ Although higher levels of physical activity are associated with reduced disease progression in $\mathrm{MCI},{ }^{73}$ it is important to know how exercise interventions delay or reverse cognitive decline in a population where activity levels may be low. Previous meta-analyses have shown both negligible ${ }^{8}$ and positive ${ }^{11}$ effects on cognitive function in populations with MCI. Although it is not clear whether exercise is more or less effective in cognitively impaired or intact patients, the results of our analysis provide additional evidence that physical exercise has a positive effect on cognitive function in patients with MCI. Second, when the control group used no contact (eg, usual care, waiting list) or an education programme (eg, health lectures, computer course), the effect estimates were all significantly beneficial in comparison with either an active or social control. This finding is of interest as it suggests that education alone is not sufficient to promote changes in lifestyle habits which can be of measurable benefit in the short term.

\section{Strengths and limitations}

A major strength of this study is that it provides an up-to-date summary of supervised RCTs of physical exercise for cognitive function in adults aged $>50$ years and advances previous and recent reviews by employing a multilevel design and not limiting exercise mode or publication date. Despite this, the findings of the review must be considered in the context of a number of limitations. First, the search strategy was limited to English language publications and thus there is a possibility of a language bias in the systematic review. Second, studies were included only if exercise was the sole intervention. Consequently, a large number of studies which used exercise as an adjunct component to another intervention (eg, combined cognitive and physical exercise; see Law $e^{~} \mathrm{al}^{74}$ ) were excluded. As the objectives of this review were to examine the effect of physical exercise, it was not appropriate to include such studies. Additionally, the RCTs included here were strictly limited to fully supervised exercise interventions. This inclusion criterion was implemented as it is important to acknowledge the methodological and conceptual differences between a supervised and unsupervised intervention. From a public health perspective, however, the effect of unsupervised exercise or a comparison between supervised and unsupervised exercise interventions would be of great value. Future meta-analyses designed to specifically answer this question are required.

\section{CONCLUSIONS}

This meta-analysis showed that physical exercise interventions are effective at improving the cognitive function of older adults, regardless of baseline cognitive status. Interventions of aerobic, resistance training, multicomponent training and tai chi were similarly effective. The findings suggest that an exercise programme with components of both aerobic and resistance-type training, of at least moderate intensity and at least $45 \mathrm{~min}$ per session, on as many days of the week as possible, is beneficial to cognitive function in adults aged $>50$ years.

\section{What are the new findings?}

- Physical exercise interventions significantly improved cognitive function in adults older than 50 years, regardless of baseline cognitive status.

- Positive benefits to cognition occurred with an exercise intervention that included tai chi, or resistance and aerobic training, prescribed either in isolation or combined.

- When exercise training variables were considered, interventions that included exercise with a minimum duration of $45 \mathrm{~min}$ and at moderate to vigorous intensity showed improvements to cognitive function.

How might it impact on clinical practice in the near future?

- This meta-analysis provides positive evidence that a combination of aerobic and resistance type exercise of at least moderate intensity on as many days of the week as feasible is beneficial to cognitive function.

- Tai chi may be a promising intervention aimed at brain health for the over 50s, although further high quality randomised controlled trials are required to confirm the benefits shown in this study.

- The dosage of physical exercise is important and clinicians should ensure their exercise recommendations are individualised and provide a sufficient training stimulus.

Acknowledgements The authors report no disclosures. NC is funded by an ARC Future Fellowship No 120100227. The authors thank Dr Marijke Welvaert for advising on the statistical methodology.

Contributors JMN contributed to the design of the study, the literature search, data screening and extraction, conducted all statistical analyses, and managed all aspects of manuscript preparation and submission. He is guarantor. NC contributed to the design of the study, provided methodological input and theoretical expertise, and contributed to writing and editing of the manuscript. KLP contributed to the design of the study, data screening and extraction, and contributed to writing and editing of the manuscript. DJS contributed to the design of the study, data screening and extraction, and contributed to writing and editing of the manuscript. BR contributed to the design of the study, data screening and extraction, provided methodological input and theoretical expertise, and contributed to writing and editing of the manuscript. All authors meet the criteria for authorship and JM Northey accepts to provide access to the data on request.

Competing interests None declared.

Provenance and peer review Not commissioned; externally peer reviewed.

(c) Article author(s) (or their employer(s) unless otherwise stated in the text of the article) 2018. All rights reserved. No commercial use is permitted unless otherwise expressly granted. 


\section{REFERENCES}

1 Prakash RS, Voss MW, Erickson KI, et al. Physical activity and cognitive vitality. Annu Rev Psychol 2015;66:769-97.

2 Erickson KI, Gildengers AG, Butters MA. Physical activity and brain plasticity in late adulthood. Dialogues Clin Neurosci 2013;15:99-108.

3 Scarmeas N, Stern Y. Cognitive reserve and lifestyle. J Clin Exp Neuropsychol 2003;25:625-33.

4 Abbott RD, White LR, Ross GW, et al. Walking and dementia in physically capable elderly men. JAMA 2004;292:1447-53.

5 Rasmussen P, Brassard P, Adser $\mathrm{H}$, et al. Evidence for a release of brain-derived neurotrophic factor from the brain during exercise. Exp Physiol 2009;94:1062-9.

6 Colcombe S, Kramer AF. Fitness effects on the cognitive function of older adults: a meta-analytic study. Psychol Sci 2003;14:125-30.

7 Snowden M, Steinman L, Mochan K, et al. Effect of exercise on cognitive performance in community-dwelling older adults: review of intervention trials and recommendations for public health practice and research. J Am Geriatr Soc 2011;59:704-16.

8 Gates N, Fiatarone Singh MA, Sachdev PS, et al. The effect of exercise training on cognitive function in older adults with mild cognitive impairment: a meta-analysis of randomized controlled trials. Am J Geriatr Psychiatry 2013:21:1086-97.

9 Kelly ME, Loughrey D, Lawlor BA, et al. The impact of exercise on the cognitive functioning of healthy older adults: a systematic review and meta-analysis. Ageing Res Rev 2014;16:12-31.

10 Young J, Angevaren M, Rusted J, et al. Aerobic exercise to improve cognitive function in older people without known cognitive impairment. Cochrane Database Syst Rev 2015:4:CD005381.

11 Zheng G, Xia R, Zhou W, et al. Aerobic exercise ameliorates cognitive function in older adults with mild cognitive impairment: a systematic review and meta-analysis of randomised controlled trials. Br J Sports Med 2016;50:1443-50.

12 Chodzko-Zajko WJ, Proctor DN, Fiatarone Singh MA, et al. Exercise and physical activity for older adults. Med Sci Sports Exerc 2009;41:1510-30.

13 WHO. Global recommendations on physical activity for health. Geneva: World Health Organization, 2010.

14 Gothe NP, McAuley E. Yoga and cognition: a meta-analysis of chronic and acute effects. Psychosom Med 2015;77:784-97.

15 Wayne PM, Walsh JN, Taylor-Piliae RE, et al. Effect of Tai Chi on cognitive performance in older adults: systematic review and meta-analysis. J Am Geriatr Soc 2014;62:25-39.

16 Moher D, Liberati A, Tetzlaff J, et al; PRISMA Group. Preferred reporting items for systematic reviews and meta-analyses: the PRISMA statement. BMJ 2009;339:b2535.

17 Colcombe SJ, Kramer AF, Erickson KI, et al. Cardiovascular fitness, cortical plasticity, and aging. Proc Natl Acad Sci U S A 2004;101:3316-21.

18 Langlois F, Vu TT, Chassé K, et al. Benefits of physical exercise training on cognition and quality of life in frail older adults. J Gerontol B Psychol Sci Soc Sci 2013;68:400-4.

19 Babaei P, Azali Alamdari K, Soltani Tehrani B, et al. Effect of six weeks of endurance exercise and following detraining on serum brain derived neurotrophic factor and memory performance in middle aged males with metabolic syndrome. J Sports Med Phys Fitness 2013;53:437-43.

20 Tsai CL, Wang CH, Pan CY, et al. The effects of long-term resistance exercise on the relationship between neurocognitive performance and GH, IGF-1, and homocysteine levels in the elderly. Front Behav Neurosci 2015;9:23.

21 Yoon DH, Kang D, Kim HJ, et al. Effect of elastic band-based high-speed power training on cognitive function, physical performance and muscle strength in older women with mild cognitive impairment. Geriatr Gerontol Int 2016.

22 ACSM. ACSM's guidelines for exercise testing and prescription: Lippincott Williams \& Wilkins, 2013

23 Norton K, Norton L, Sadgrove D. Position statement on physical activity and exercise intensity terminology. J Sci Med Sport 2010;13:496-502.

24 Smith PJ, Blumenthal JA, Hoffman BM, et al. Aerobic exercise and neurocognitive performance: a meta-analytic review of randomized controlled trials. Psychosom Med 2010;72:239-52.

25 Higgins J, Green S, The Cochrane Collaboration. Cochrane handbook for systematic reviews of interventions. NY: John Wiley \& Sons, 2011.

26 R Core Team. R: a language and environment for statistical computing. Vienna, Austria: R foundation for statistical computing, 2012. ISBN 3-900051-07-0, 2014.

27 Viechtbauer W. Conducting meta-analyses in $R$ with the metafor package. J Stat Softw 2010;36:1-48.

28 Weisz JR, Kuppens S, Eckshtain D, et al. Performance of evidence-based youth psychotherapies compared with usual clinical care: a multilevel meta-analysis. JAMA Psychiatry 2013;70:750-61.

29 Higgins JP, Thompson SG, Deeks JJ, et al. Measuring inconsistency in meta-analyses. BMJ 2003;327:557-60

30 Egger M, Davey Smith G, Schneider M, et al. Bias in meta-analysis detected by a simple, graphical test. BMJ 1997;315:629-34.

31 Habeck CW, Schultz AK. Community-level impacts of white-tailed deer on understorey plants in north American forests: a meta-analysis. AoB Plants 2015;7:plv119.
32 Sterne JA, Sutton AJ, loannidis JP, et al. Recommendations for examining and interpreting funnel plot asymmetry in meta-analyses of randomised controlled trials. BMJ 2011;343:d4002.

33 Atkins D, Best D, Briss PA, Oxman A, et al; GRADE Working Group. Grading quality of evidence and strength of recommendations. BMJ 2004;328:1490.

34 Blumenthal JA, Emery CF, Madden DJ, et al. Cardiovascular and behavioral effects of aerobic exercise training in healthy older men and women. J Gerontol 1989:44:M147-M157.

35 Madden DJ, Blumenthal JA, Allen PA, et al. Improving aerobic capacity in healthy olde adults does not necessarily lead to improved cognitive performance. Psychol Aging 1989;4:307-20.

36 Liu-Ambrose T, Nagamatsu LS, Graf P, et al. Resistance training and executive functions: a 12-month randomized controlled trial. Arch Intern Med 2010;170:170-8.

37 Liu-Ambrose T, Nagamatsu LS, Voss MW, et al. Resistance training and functional plasticity of the aging brain: a 12-month randomized controlled trial. Neurobiol Aging 2012;33:1690-8.

38 Nagamatsu LS, Handy TC, Hsu CL, et al. Resistance training promotes cognitive and functional brain plasticity in seniors with probable mild cognitive impairment. Arch Intern Med 2012;172:666-8.

39 ten Brinke LF, Bolandzadeh N, Nagamatsu LS, et al. Aerobic exercise increases hippocampal volume in older women with probable mild cognitive impairment: a 6-month randomised controlled trial. Br J Sports Med 2015;49:248-54.

40 Erickson $\mathrm{Kl}$, Voss MW, Prakash RS, et al. Exercise training increases size of hippocampus and improves memory. Proc Natl Acad Sci U S A 2011;108:3017-22.

41 Voss MW, Heo S, Prakash RS, et al. The influence of aerobic fitness on cerebral white matter integrity and cognitive function in older adults: results of a one-year exercise intervention. Hum Brain Mapp 2013;34:2972-85.

42 Albinet $\mathrm{CT}$, Boucard G, Bouquet CA, et al. Increased heart rate variability and executive performance after aerobic training in the elderly. Eur J Appl Physiol 2010;109:617-24.

43 Emery CF, Gatz M. Psychological and cognitive effects of an exercise program for community-residing older adults. Gerontologist 1990;30:184-8.

44 Fabre C, Chamari K, Mucci P, et al. Improvement of cognitive function by mental and/or individualized aerobic training in healthy elderly subjects. Int J Sports Med 2002;23:415-21.

45 Hawkins HL, Kramer AF, Capaldi D. Aging, exercise, and attention. Psychol Aging 1992; 7:643-53.

46 Mortimer JA, Ding D, Borenstein AR, et al. Changes in brain volume and cognition in a randomized trial of exercise and social interaction in a community-based sample of non-demented Chinese elders. J Alzheimers Dis 2012;30:757-66.

47 Moul JL, Goldman B, Warren B. Physical activity and cognitive performance in the older population. J Aging Phys Act 1995;3:135-45.

48 Muscari A, Giannoni C, Pierpaoli L, et al. Chronic endurance exercise training prevents aging-related cognitive decline in healthy older adults: a randomized controlled trial. Int J Geriatr Psychiatry 2010;25:1055-64.

49 Whitehurst M. Reaction time unchanged in older women following aerobic training Percept Mot Skills 1991:72:251-6.

50 Albinet CT, Abou-Dest A, André N, et al. Executive functions improvement following a 5-month aquaerobics program in older adults: role of cardiac vagal control in inhibition performance. Biol Psychol 2016;115:69-77.

51 Antunes HK, De Mello MT, Santos-Galduróz RF, et al. Effects of a physical fitness program on memory and blood viscosity in sedentary elderly men. Braz J Med Biol Res 2015;48:805-12

52 Antunes HKM, Santos-Galduroz RF, De Aquino Lemos V, et al. The influence of physica exercise and leisure activity on neuropsychological functioning in older adults. Age 2015:37:9815

53 Busse A, Jacob Filho W, Magaldi R, et al. Effects of resistance training exercise on cognitive performance in elderly individuals with memory impairment: results of a controlled trial. Einstein 2008;6:402-7.

54 Cassilhas RC, Viana VA, Grassmann V, et al. The impact of resistance exercise on the cognitive function of the elderly. Med Sci Sports Exerc 2007;39:1401-7.

55 Kimura K, Obuchi S, Arai T, et al. The influence of short-term strength training on health-related quality of life and executive cognitive function. J Physiol Anthropol 2010:29:95-101.

56 Tsutsumi T, Don BM, Zaichkowsky LD, et al. Physical fitness and psychological benefits of strength training in community dwelling older adults. App/ Human Sci 1997; 16:257-66.

57 Ansai JH, Rebelatto JR. Effect of two physical exercise protocols on cognition and depressive symptoms in oldest-old people: a randomized controlled trial. Geriatr Gerontol Int 2015:15:1127-34.

58 Fragala MS, Beyer KS, Jajtner AR, et al. Resistance exercise may improve spatial awareness and visual reaction in older adults. J Strength Cond Res 2014;28:2079-87.

59 Fiatarone Singh MA, Gates N, Saigal N, et al. The Study of Mental and Resistance Training (SMART) study — resistance training and/or cognitive training in mild cognitive impairment: a randomized, double-blind, double-sham controlled trial. J Am Med Dir Assoc 2014;15:873-80 
60 Barnes DE, Santos-Modesitt W, Poelke G, et al. The Mental Activity and eXercise (MAX) trial: a randomized controlled trial to enhance cognitive function in older adults. JAMA Intern Med 2013;173:797-804.

61 Klusmann V, Evers A, Schwarzer R, et al. Complex mental and physical activity in older women and cognitive performance: a 6-month randomized controlled trial. J Gerontol A Biol Sci Med Sci 2010;65:680-8.

62 Okumiya K, Matsubayashi K, Wada T, et al. Effects of exercise on neurobehavioral function in community-dwelling older people more than 75 years of age. J Am Geriatr Soc 1996;44:569-72

63 Williams P, Lord SR. Effects of group exercise on cognitive functioning and mood in older women. Aust N Z J Public Health 1997:21:45-52.

64 Linde K, Alfermann D. Single versus combined cognitive and physical activity effects on fluid cognitive abilities of healthy older adults: a 4-month randomized controlled trial with follow-up. J Aging Phys Act 2014;22:302-13.

65 Napoli N, Shah K, Waters DL, et al. Effect of weight loss, exercise, or both on cognition and quality of life in obese older adults. Am J Clin Nutr 2014;100:189-98.

66 León J, Ureña A, Bolaños MJ, et al. A combination of physical and cognitive exercise improves reaction time in persons 61-84 years old. J Aging Phys Act 2015;23:72-7.

67 Nguyen MH, Kruse A. A randomized controlled trial of Tai chi for balance, sleep quality and cognitive performance in elderly Vietnamese. Clin Interv Aging 2012;7:185-90.
68 Tsai PF, Chang JY, Beck C, et al. A pilot cluster-randomized trial of a 20-week Tai Chi program in elders with cognitive impairment and osteoarthritic knee: effects on pain and other health outcomes. J Pain Symptom Manage 2013;45:660-9.

69 Tao J, Liu J, Egorova N, et al. Increased hippocampus-medial prefrontal cortex restingstate functional connectivity and memory function after tai chi chuan practice in elder adults. Front Aging Neurosci 2016;8:25.

70 Gothe NP, Kramer AF, McAuley E. The effects of an 8-week Hatha yoga intervention on executive function in older adults. J Gerontol A Biol Sci Med Sci 2014;69:1109-16.

71 Lucas SJ, Cotter JD, Brassard P, et al. High-intensity interval exercise and cerebrovascular health: curiosity, cause, and consequence. J Cereb Blood Flow Metab 2015;35:902-11.

72 Petersen RC, Smith GE, Waring SC, et al. Mild cognitive impairment: clinical characterization and outcome. Arch Neurol 1999;56:303-8.

73 Burns JM, Cronk BB, Anderson HS, et al. Cardiorespiratory fitness and brain atrophy in early Alzheimer disease. Neurology 2008;71:210-6.

74 Law LL, Barnett F, Yau MK, et al. Effects of combined cognitive and exercise interventions on cognition in older adults with and without cognitive impairment: a systematic review. Ageing Res Rev 2014;15:61-75. 